\title{
Tiling Space by Platonic Solids, I*
}

\author{
O. Delgado Friedrichs and D. H. Huson \\ FSP Mathematisierung, Bielefeld University, \\ 33501 Bielefeld, Germany \\ \{delgado, huson\}@mathematik.uni-bielefeld.de
}

\begin{abstract}
There exist precisely 914, 58, and 46 equivariant types of tile-transitive tilings of three-dimensional euclidean space by topological cubes, octahedra, and tetrahedra, that fall into 11,3, and 9 topological families, respectively. Representatives are described for all topological families. A general method for obtaining results of this kind is introduced.
\end{abstract}

\section{Introduction}

Of the five regular platonic solids, only the cube tiles euclidean space, and it does so in precisely one way (requiring, as we shall, that tilings be face-to-face). What is the situation if we consider tile-transitive tilings by topologically platonic solids, i.e., by topological polyhedra that are homeomorphic to one of the platonic solids, but possibly less symmetric?

We discuss this question in a series of two papers. In this, the first paper, we state that there exist precisely 914,58 , and 46 equivariant types of tile-transitive tilings of euclidean space by topological cubes, octahedra, and tetrahedra, in 11,3, and 9 topological families, respectively. In a second paper we will discuss the dodecahedron and icosahedron.

In Section 2 we recall some basic definitions. This is followed by a presentation of the classification results in Section 3. We give a detailed description of each of the topological families in Figs. 1-23, including straight-edge representatives for 22 of the 23 families. (It can be shown that the topological type CT8 does not possess a representative with straight edges.) Moreover, we determine precisely which convex tetrahedra give rise to tile-transitive tilings. Finally, in Section 4 we introduce a general method for obtaining results of this kind, the main step of which was recently developed by the first author [De].

\footnotetext{
* The second author was supported in part by the Deutsche Forschungsgemeinschaft.
} 
This work generalizes earlier methods and confirms previous results obtained in a number of special cases [DHM],[MP1],[M], [P], [MP2].

The enumeration presented here is just a first example of the results obtainable by our approach. Our method is by no means restricted to platonic solids, i.e., in principle it can be used for any type of three-dimensional topological polyhedron, and it is not restricted to tile-transitive tilings, i.e., it can be easily adapted to enumerate tilings with more than one class of tiles, as we will discuss in a forthcoming paper.

Finally, note that results of this kind have applications in structural chemistry. For example, by dualization, representatives of each of the nine topological types of tiletransitive tilings by tetrahedra give rise to a vertex-transitive tiling with tetrahedral vertices. Interpreting each vertex as a 4-valent "T-atom" and each edge as a "T-O-T-bond," these tilings correspond to zeolite structures [Sm].

\section{Definitions}

Let $\mathcal{X}$ be a simply connected manifold. A system $(\mathcal{T}, \Gamma)$, consisting of a tiling $\mathcal{T}$ of $\mathcal{X}$ and a group of homeomorphisms $\Gamma$ of $\mathcal{X}$ with $\Gamma(\mathcal{T}):=\{\gamma T \mid \gamma \in \Gamma, T \in \mathcal{T}\}=\mathcal{T}$, is called an (equivariant) tiling. We call $(\mathcal{T}, \Gamma)$ tile-transitive if $\Gamma$ acts transitively on $\mathcal{T}$, and fundamental if any $P \in \mathcal{T}$ is a fundamental domain for $\Gamma$.

Two such tilings $(\mathcal{T}, \Gamma)$ and $\left(\mathcal{T}^{\prime}, \Gamma^{\prime}\right)$ belong to the same topological family, are of the same topological type, or are topologically equivalent, if there exists a homeomorphism $\varphi: \mathcal{X} \rightarrow \mathcal{X}$ with $\varphi(\mathcal{T})=\mathcal{T}^{\prime}$. They are equivariantly equivalent if, additionally, $\Gamma^{\prime}=$ $\varphi \Gamma \varphi^{-1}$ holds, and we say that $\left(\mathcal{T}^{\prime}, \Gamma^{\prime}\right)$ is obtained from $(\mathcal{T}, \Gamma)$ by symmetry breaking if we have $\Gamma^{\prime} \leq \varphi \Gamma \varphi^{-1}$. A tiling $(\mathcal{T}, \Gamma)$ that cannot be obtained from some other tiling by symmetry breaking is called maximal.

In this paper we consider three-dimensional euclidean equivariant tilings $(\mathcal{T}, \Gamma)$, where $\mathcal{T}$ is a face-to-face tiling of euclidean space $\mathbb{E}^{3}$, and $\Gamma$ is a crystallographic space group, i.e., a discrete group of isometries with compact fundamental domain.

For such tilings, the following useful and nontrivial statement can be proved (see Section 4.2 below): Two maximal tilings are equivariantly equivalent if and only if they are topologically equivalent. Hence, each equivariant type of maximal tiling represents a topological family.

Moreover, all tilings considered are polyhedral in the sense that each tile is a topological polyhedron, i.e., a topological ball whose surface is tiled by a finite set of twodimensional faces with the usual properties. In particular, by a topological cube we mean a topological polyhedron whose edge skeleton is isomorphic to the edge skeleton of a regular cube, etc.

\section{The Classification}

In this section we present our results. First we state the three main theorems.

Theorem 3.1. There exist precisely 11 topological types and 914 equivariant types of tile-transitive tilings of three-dimensional euclidean space by topological cubes. The topological types are described in Figs. 1-11. 
Theorem 3.2. There exist precisely 3 topological types and 58 equivariant types of tile-transitive tilings of three-dimensional euclidean space by topological octahedra. The topological types are described in Figs. 12-14.

Theorem 3.3. There exist precisely 9 topological types and 46 equivariant types of tile-transitive tilings of three-dimensional euclidean space by topological tetrahedra. The topological types are described in Figs. 15-23.

Details of the classification into topological families are given in Figs. 1-23.

In each figure, we depict a patch of tiles from a straight-edge representative $(\mathcal{T}, \Gamma)$ of the given topological family (except CT8). Tiles are shrunk slightly. Each such picture is accompanied by a diagram that gives a precise description of the tiling $(\mathcal{T}, \Gamma)$.

First we note that each such diagram gives a description of a tile $T$ and its stabilizer group $\Gamma_{T}:=\{\gamma \in \Gamma \mid \gamma(T)=T\}$. To be precise, each diagram is based on the Schlegel diagram representing the combinatorial or topological structure of a tile $T \in \mathcal{T}$. A gray polygon, if present, represents a fundamental domain for $\Gamma_{T}$. If no such gray polygon is present, then $\Gamma_{T}$ consists of the identity only and the fundamental domain consists of the whole tile $T$. (As we are considering tile-transitive tilings, a fundamental domain for $\Gamma_{T}$ corresponds to a fundamental domain for $\Gamma$.) Each such gray polygon contains a small circle $\circ$ which specifies a flag of the tile, i.e., a triple $(v, e, f)$ consisting of a vertex $v$, an edge $e$, and a face $f$, with $v \subset e \subset f$. Additional circles indicate the $\Gamma_{T}$-orbit of $\circ$ and thus unambiguously define the action of $\Gamma_{T}$ on $T$.

Second, further abstract generators for the symmetry group $\Gamma$ can be obtained from the diagram as follows. The generators are given by adjacency transformations defined by admissible face pairings. Two faces form an admissible pair, if they are both marked by the same capital letter $A, B, C, \ldots$ and precisely one carries a plus sign, the other one a minus sign, e.g., $A+$ and $A-$. Note that the position of the letter is important, as it corresponds to a flag (as above), and the pairing is understood to map letter onto letter (i.e., flag onto flag). A face that does not contain a letter is paired with itself by the identity pairing.

Moreover, a set of defining relations for the group $\Gamma$ can be obtained in a straightforward manner, similar to the method described on p. 160 of [V].

Below each diagram we state: Group: the crystallographic name of the symmetry group [Hah], Stab.: the stabilizer group of a tile $T$ in orbifold notation [C] (see Table 1),

Table 1. Conway's orbifold notation (i) and standard crystallographic notation (ii) for the crystallographic point-groups.

\begin{tabular}{cccccccc}
\hline (i) & (ii) & (i) & (ii) & (i) & (ii) & (i) & (ii) \\
\hline 1 & 1 & $2 \times$ & $\overline{4}$ & 432 & 432 & $* 322$ & $\overline{6} 2 m$ \\
$1 *$ & $m$ & 322 & 32 & 44 & 4 & $* 33$ & $3 m$ \\
$1 \times$ & $\overline{1}$ & 33 & 3 & $4 *$ & $4 / m$ & $* 332$ & $\overline{4} 3 m$ \\
22 & 2 & 332 & 23 & 622 & 622 & $* 422$ & $4 / m m m$ \\
222 & 222 & $3 *$ & $3 / m$ & 66 & 6 & $* 432$ & $m \overline{3} m$ \\
$2 *$ & $2 / m$ & $3 * 2$ & $m \overline{3}$ & $6 *$ & $6 / m$ & $* 44$ & $4 m m$ \\
$2 * 2$ & $\overline{4} 2 m$ & $3 \times$ & $\overline{3}$ & $* 22$ & $m m 2$ & $* 622$ & $6 / \mathrm{mmm}$ \\
$2 * 3$ & $\overline{3} m$ & 422 & 422 & $* 222$ & $m m m$ & $* 66$ & $6 m m$ \\
\hline
\end{tabular}



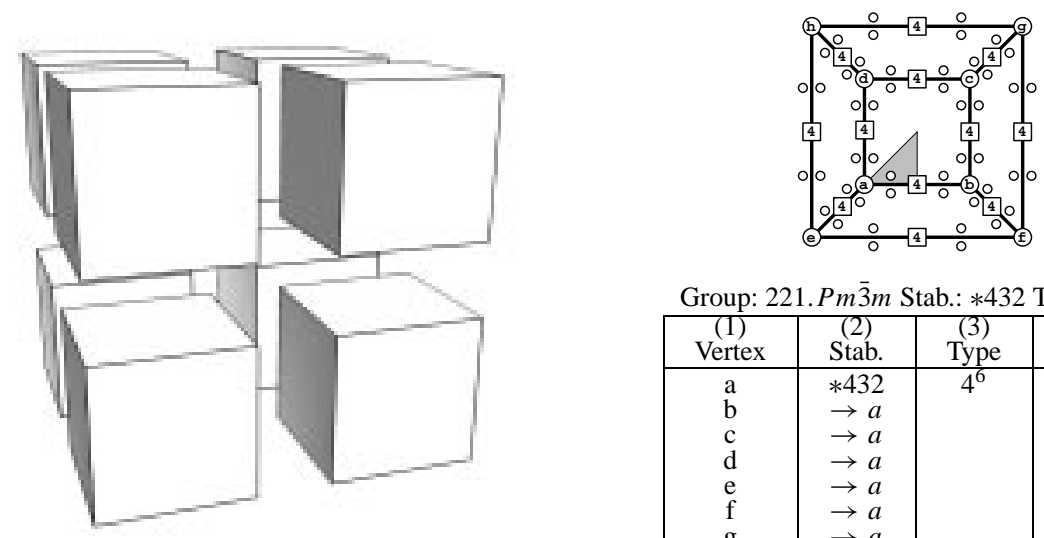

Group: $221 . P m \overline{3} m$ Stab.: $* 432$ Types: 786
\begin{tabular}{|c|c|c|c|}
\hline (1) & $(2)$ & $(3)$ & $(4)$ \\
Vertex & Stab. & Type & Coord. \\
\hline $\mathrm{a}$ & $* 432$ & $4^{6}$ & $0,0,0$ \\
$\mathrm{~b}$ & $\rightarrow a$ & & $1,0,0$ \\
$\mathrm{c}$ & $\rightarrow a$ & & $1,1,0$ \\
$\mathrm{~d}$ & $\rightarrow a$ & & $0,1,0$ \\
$\mathrm{e}$ & $\rightarrow a$ & & $0,0,1$ \\
$\mathrm{f}$ & $\rightarrow a$ & & $1,0,1$ \\
$\mathrm{~g}$ & $\rightarrow a$ & & $1,1,1$ \\
$\mathrm{~h}$ & $\rightarrow a$ & & $0,1,1$ \\
\hline
\end{tabular}

Fig. 1. CT1.
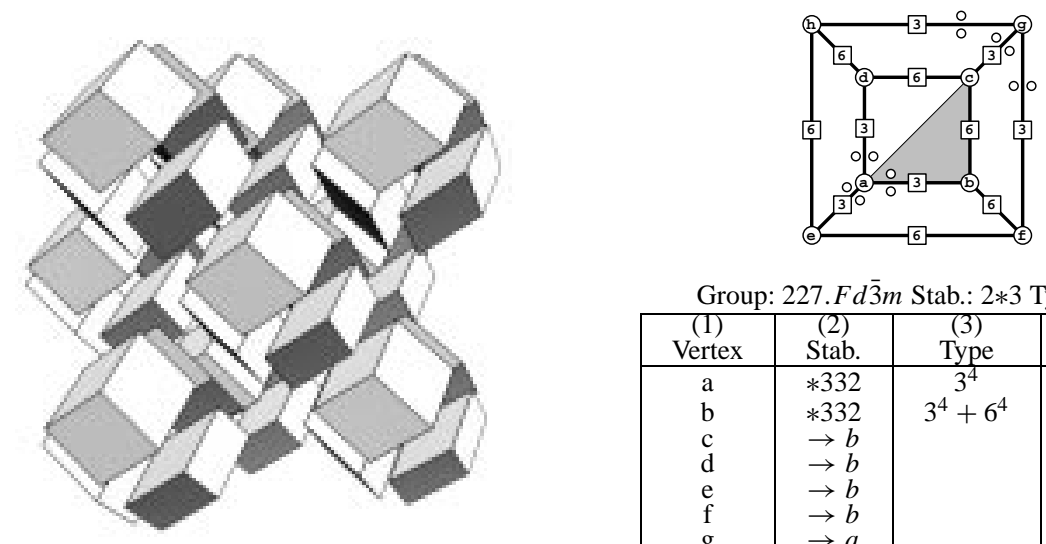

Group: $227 . F d \overline{3} m$ Stab.: $2 * 3$ Types: 18

\begin{tabular}{|c|c|c|c|}
\hline Vertex & Stab. & $\begin{array}{c}(3) \\
\text { Type }\end{array}$ & $\left.\begin{array}{c}\text { Coord. } \\
\hline \mathrm{a}\end{array} *^{4}\right)$ \\
$\mathrm{b}$ & $* 332$ & $3^{4}$ & $0,0,0$ \\
$\mathrm{c}$ & $\rightarrow b$ & $3^{4}+6^{4}$ & $-1,1,1$ \\
$\mathrm{~d}$ & $\rightarrow b$ & & $0,2,0$ \\
$\mathrm{e}$ & $\rightarrow b$ & & $1,1,-1$ \\
$\mathrm{f}$ & $\rightarrow b$ & & $1,-1,1$ \\
$\mathrm{~g}$ & $\rightarrow a$ & & $0,0,2$ \\
$\mathrm{~h}$ & $\rightarrow b$ & & $1,1,1$ \\
\hline
\end{tabular}

Fig. 2. CT2.
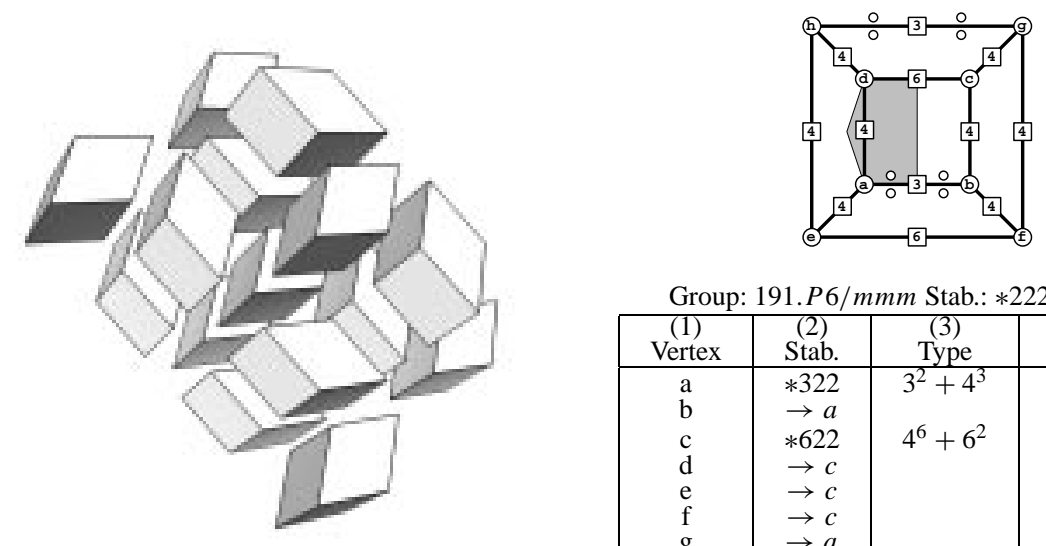

Group: 191.P6/mmm Stab.: *222 Types: 60

\begin{tabular}{|c|c|c|c|}
\hline $\begin{array}{c}(1) \\
\text { Vertex }\end{array}$ & $\begin{array}{c}(2) \\
\text { Stab. }\end{array}$ & $\begin{array}{c}\text { (3) } \\
\text { Type }\end{array}$ & $\begin{array}{c}\text { Coord. } \\
\text { Cyper }\end{array}$ \\
\hline $\mathrm{a}$ & $* 322$ & $3^{2}+4^{3}$ & $0,0,0$ \\
$\mathrm{~b}$ & $\rightarrow a$ & & $-1,-1,-1$ \\
$\mathrm{c}$ & $* 622$ & $4^{6}+6^{2}$ & $-1,-2,0$ \\
$\mathrm{~d}$ & $\rightarrow c$ & & $0,-1,1$ \\
$\mathrm{e}$ & $\rightarrow c$ & & $1,0,-1$ \\
$\mathrm{f}$ & $\rightarrow c$ & & $0,-1,-2$ \\
$\mathrm{~g}$ & $\rightarrow a$ & & $0,-2,-1$ \\
$\mathrm{~h}$ & $\rightarrow a$ & & $1,-1,0$ \\
\hline
\end{tabular}

Fig. 3. CT3. 

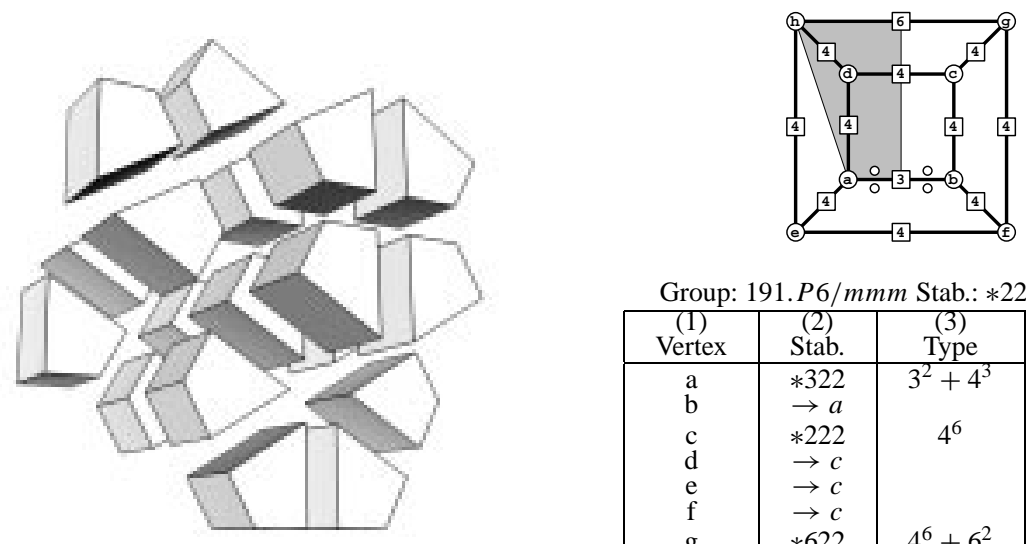

Group: $191 . P 6 / \mathrm{mmm}$ Stab.: $* 22$ Types: 31
\begin{tabular}{|c|c|c|c|}
\hline$(1)$ & $(2)$ & $(3)$ & $(4)$ \\
Vertex & Stab. & Type & Coord. \\
\hline $\mathrm{a}$ & $* 322$ & $3^{2}+4^{3}$ & $0,0,0$ \\
$\mathrm{~b}$ & $\rightarrow a$ & & $1,1,1$ \\
$\mathrm{c}$ & $* 222$ & $4^{6}$ & $3,1,-1$ \\
$\mathrm{~d}$ & $\rightarrow c$ & & $2,0,-2$ \\
$\mathrm{e}$ & $\rightarrow c$ & & $0,-2,2$ \\
$\mathrm{f}$ & $\rightarrow c$ & & $1,-1,3$ \\
$\mathrm{~g}$ & $* 622$ & $4^{6}+6^{2}$ & $5,-3,1$ \\
$\mathrm{~h}$ & $\rightarrow g$ & & $4,-4,0$ \\
\hline
\end{tabular}

Fig. 4. CT4.
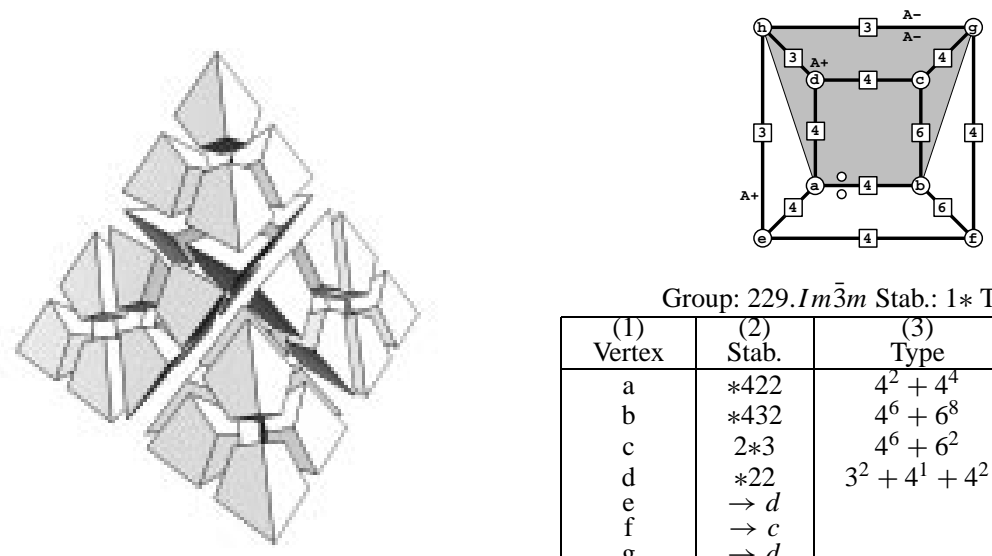

\begin{tabular}{|c|c|c|c|}
\multicolumn{5}{|c}{ Group: $229 . \operatorname{Im} \overline{3} m$ Stab.: $1 *$ Types: 5} \\
\hline Vertex & Stab. & Type & Coord. \\
\hline $\mathrm{a}$ & $* 422$ & $4^{2}+4^{4}$ & $1,-1,1$ \\
$\mathrm{~b}$ & $* 432$ & $4^{6}+6^{8}$ & $1,0,1$ \\
$\mathrm{c}$ & $2 * 3$ & $4^{6}+6^{2}$ & $1,1,1$ \\
$\mathrm{~d}$ & $* 22$ & $3^{2}+4^{1}+4^{2}$ & $0,0,0$ \\
$\mathrm{e}$ & $\rightarrow d$ & & $1,-1,2$ \\
$\mathrm{f}$ & $\rightarrow c$ & & $1,0,2$ \\
$\mathrm{~g}$ & $\rightarrow d$ & & $1,1,2$ \\
$\mathrm{~h}$ & $2 * 2$ & $3^{4}$ & $0,0,2$ \\
\hline
\end{tabular}

Fig. 5. CT5.
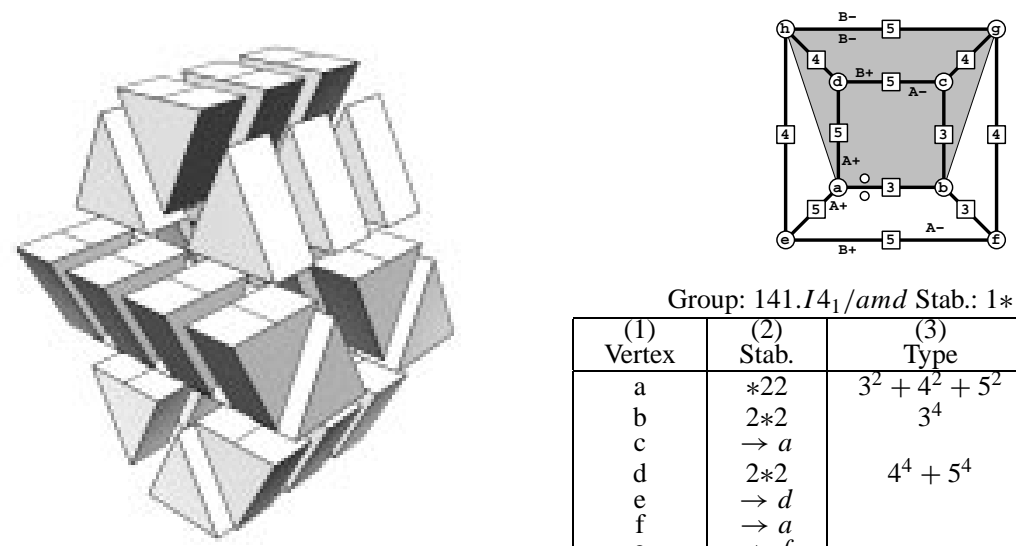

Group: 141.I41/amd Stab.: $1 *$ Types: 5

\begin{tabular}{|c|c|c|c|}
\hline $\begin{array}{c}(1) \\
\text { Vertex }\end{array}$ & $\begin{array}{l}\text { (2) } \\
\text { Stab. }\end{array}$ & $\begin{array}{l}\text { (3) } \\
\text { Type }\end{array}$ & $\begin{array}{l}\text { (4) } \\
\text { Coord. }\end{array}$ \\
\hline a & $* 22$ & $3^{2}+4^{2}+5^{2}$ & $0,1,2$ \\
\hline b & $2 * 2$ & $3^{4}$ & $0,0,2$ \\
\hline $\mathrm{c}$ & $\rightarrow a$ & & $0,-1,2$ \\
\hline $\mathrm{d}$ & $2 * 2$ & $4^{4}+5^{4}$ & $0,0,0$ \\
\hline $\begin{array}{l}e \\
f\end{array}$ & $\rightarrow d$ & & $-1,1,2$ \\
\hline $\begin{array}{l}\mathrm{f} \\
\mathrm{g}\end{array}$ & $\begin{array}{l}\rightarrow a \\
\rightarrow f\end{array}$ & & $-1,-1,2$ \\
\hline $\mathrm{h}$ & $\rightarrow d$ & & $-1,0,0$ \\
\hline
\end{tabular}

Fig. 6. CT6. 

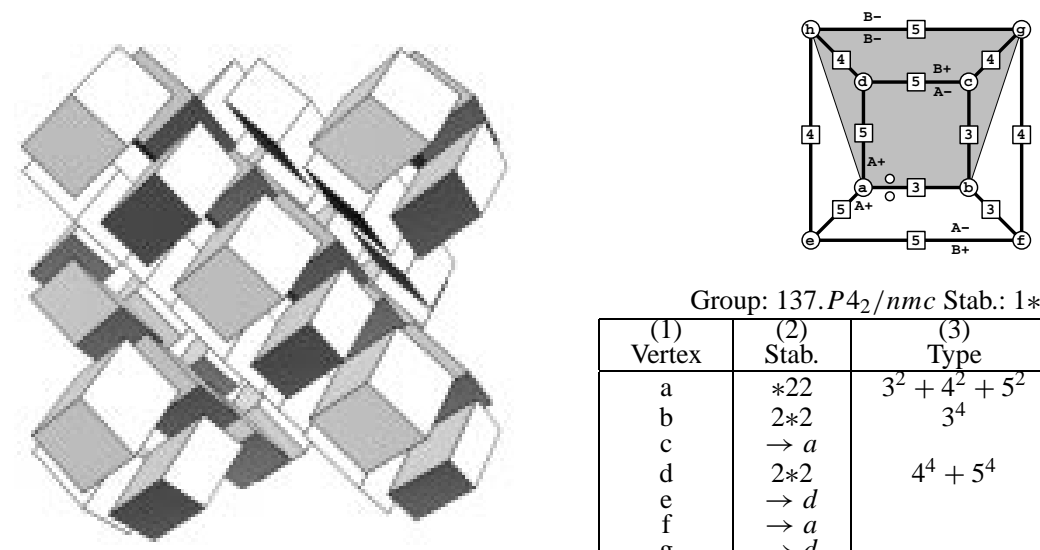

Group: $137 . P 4_{2} / n m c$ Stab.: $1 *$ Types: 5
\begin{tabular}{|c|c|c|c|}
\hline$(1)$ & $(2)$ & $(3)$ & $(4)$ \\
Vertex & Stab. & Type & Coord. \\
\hline a & $* 22$ & $3^{2}+4^{2}+5^{2}$ & $1,1,1$ \\
b & $2 * 2$ & $3^{4}$ & $0,0,2$ \\
c & $\rightarrow a$ & & $-1,-1,1$ \\
d & $2 * 2$ & $4^{4}+5^{4}$ & $0,0,0$ \\
e & $\rightarrow d$ & & $2,0,0$ \\
$\mathrm{f}$ & $\rightarrow a$ & & $1,-1,1$ \\
g & $\rightarrow d$ & & $0,-2,0$ \\
h & $\rightarrow a$ & & $1,-1,-1$ \\
\hline
\end{tabular}

Fig. 7. CT7.

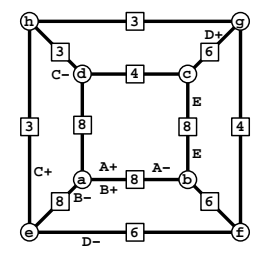

Group: 142.I41/acd Stab.: 1 Types: 1

\begin{tabular}{|c|c|c|c|}
\hline $\begin{array}{c}(1) \\
\text { Vertex }\end{array}$ & $\begin{array}{c}(2) \\
\text { Stab. }\end{array}$ & Type & Coord. \\
\hline $\mathrm{a}$ & 222 & $3^{4}+4^{4}+6^{4}+8^{4}$ & - \\
$\mathrm{b}$ & $\rightarrow a$ & & - \\
$\mathrm{c}$ & $\rightarrow a$ & & - \\
$\mathrm{d}$ & $\rightarrow a$ & & - \\
$\mathrm{e}$ & $\rightarrow a$ & & - \\
$\mathrm{f}$ & $\rightarrow a$ & & - \\
$\mathrm{g}$ & $\rightarrow a$ & 3 & - \\
$\mathrm{h}$ & $2 x$ & 3 & \\
\hline
\end{tabular}

Fig. 8. CT8.
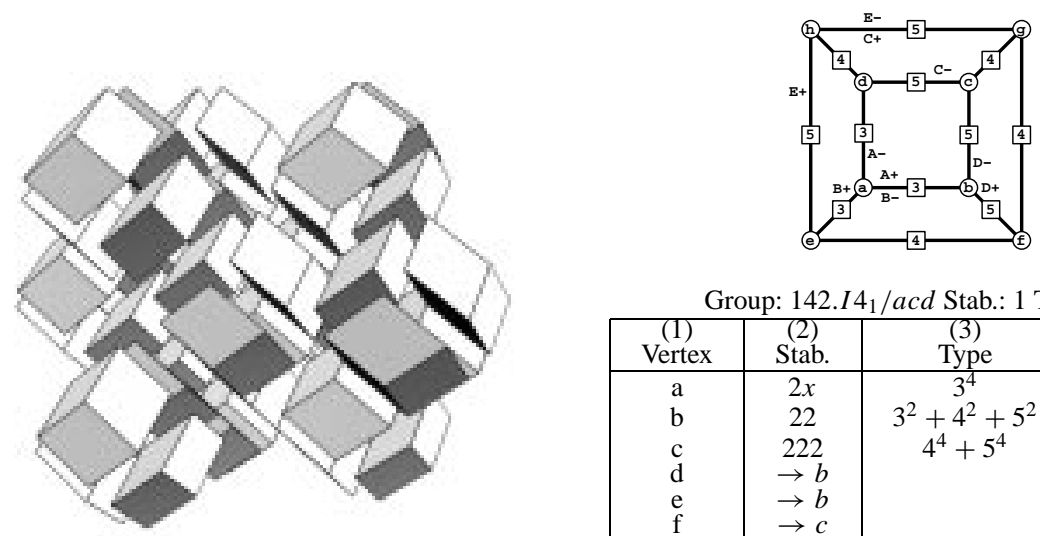

Group: $142 . I 4_{1}$ /acd Stab.: 1 Types: 1
\begin{tabular}{|c|c|c|c|}
\hline$(1)$ & $(2)$ & $(3)$ & $(4)$ \\
Vertex & Stab. & Type & Coord. \\
\hline $\mathrm{a}$ & $2 x$ & $3^{4}$ & $0,0,0$ \\
$\mathrm{~b}$ & 22 & $3^{2}+4^{2}+5^{2}$ & $1,0,1$ \\
$\mathrm{c}$ & 222 & $4^{4}+5^{4}$ & $2,0,0$ \\
$\mathrm{~d}$ & $\rightarrow b$ & & $1,0,-1$ \\
$\mathrm{e}$ & $\rightarrow b$ & & $0,1,1$ \\
$\mathrm{f}$ & $\rightarrow c$ & & $1,1,2$ \\
$\mathrm{~g}$ & $\rightarrow b$ & & $2,1,1$ \\
$\mathrm{~h}$ & $\rightarrow c$ & & $1,1,0$ \\
\hline
\end{tabular}

Fig. 9. CT9. 

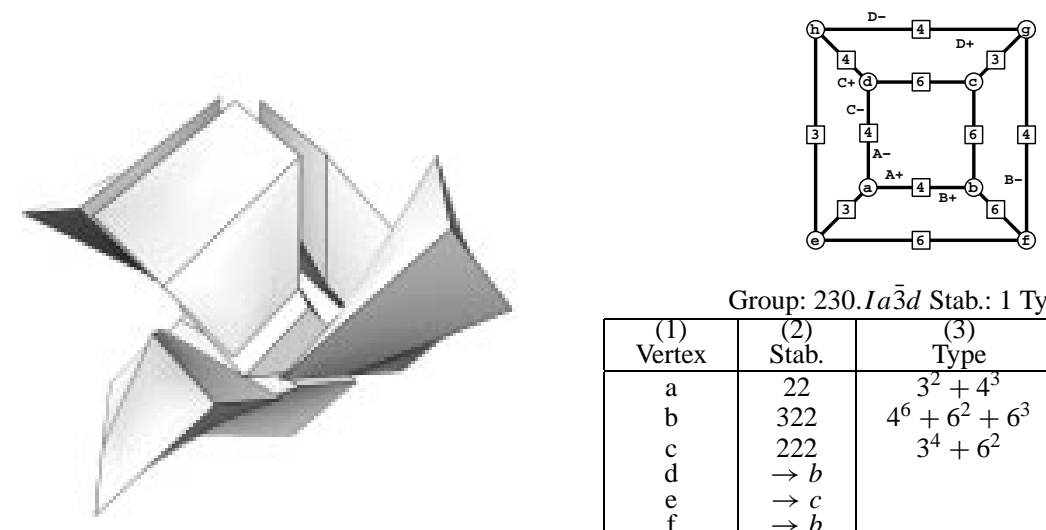

\begin{tabular}{|c|c|c|c|}
\multicolumn{5}{c|}{ Group: $230 . I \overline{3} d$ Stab.: 1 Types: 1} \\
\hline $\begin{array}{c}\text { (1) } \\
\text { Vertex }\end{array}$ & $\begin{array}{c}(2) \\
\text { Stab. }\end{array}$ & $\begin{array}{c}(3) \\
\text { Type }\end{array}$ & $\begin{array}{c}(4) \\
\text { Coord. }\end{array}$ \\
\hline $\mathrm{a}$ & 22 & $3^{2}+4^{3}$ & $0,0,0$ \\
$\mathrm{~b}$ & 322 & $4^{6}+6^{2}+6^{3}$ & $1,-1,1$ \\
$\mathrm{c}$ & 222 & $3^{4}+6^{2}$ & $3,-3,0$ \\
$\mathrm{~d}$ & $\rightarrow b$ & & $1,-1,-1$ \\
$\mathrm{e}$ & $\rightarrow c$ & & $1,0,1$ \\
$\mathrm{f}$ & $\rightarrow b$ & & $2,0,2$ \\
$\mathrm{~g}$ & $\rightarrow a$ & & $4,-2,0$ \\
$\mathrm{~h}$ & $\rightarrow a$ & & $3,-1,-1$ \\
\hline
\end{tabular}

Fig. 10. CT10.
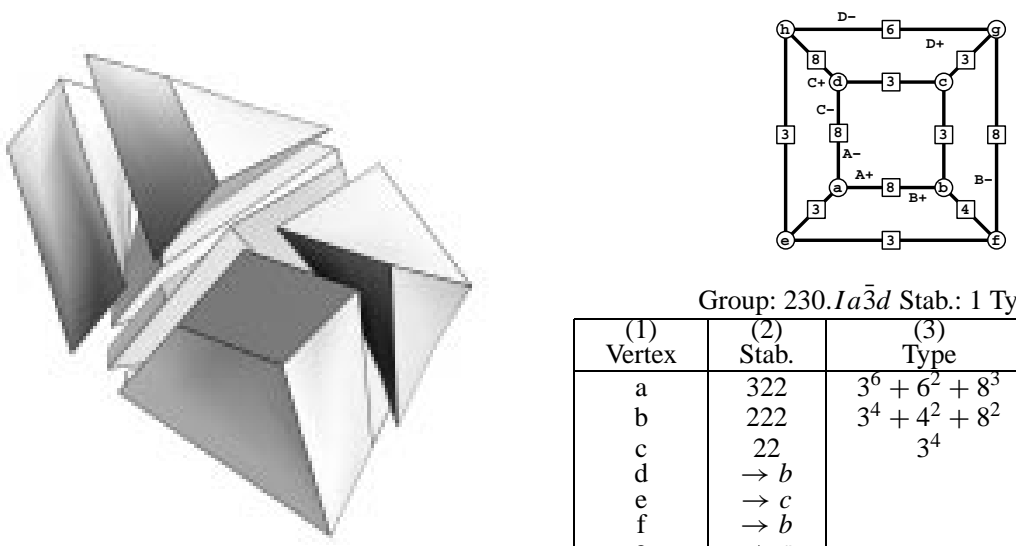

\begin{tabular}{|c|c|c|c|}
\multicolumn{5}{|c}{ Group: $230 . \operatorname{Ia} \overline{3} d$ Stab.: 1 Types: 1} \\
\hline (1) & (2) & (3) & (4) \\
Vertex & Stab. & Type & Coord. \\
\hline a & 322 & $3^{6}+6^{2}+8^{3}$ & $0,0,0$ \\
$\mathrm{~b}$ & 222 & $3^{4}+4^{2}+8^{2}$ & $2,0,2$ \\
$\mathrm{c}$ & 22 & $3^{4}$ & $5,-5,0$ \\
$\mathrm{~d}$ & $\rightarrow b$ & & $0,-2,-2$ \\
$\mathrm{e}$ & $\rightarrow c$ & & $3,0,1$ \\
$\mathrm{f}$ & $\rightarrow b$ & & $4,0,4$ \\
$\mathrm{~g}$ & $\rightarrow a$ & & $8,-4,0$ \\
$\mathrm{~h}$ & $\rightarrow a$ & & $8,-2,-2$ \\
\hline
\end{tabular}

Fig. 11. CT11.
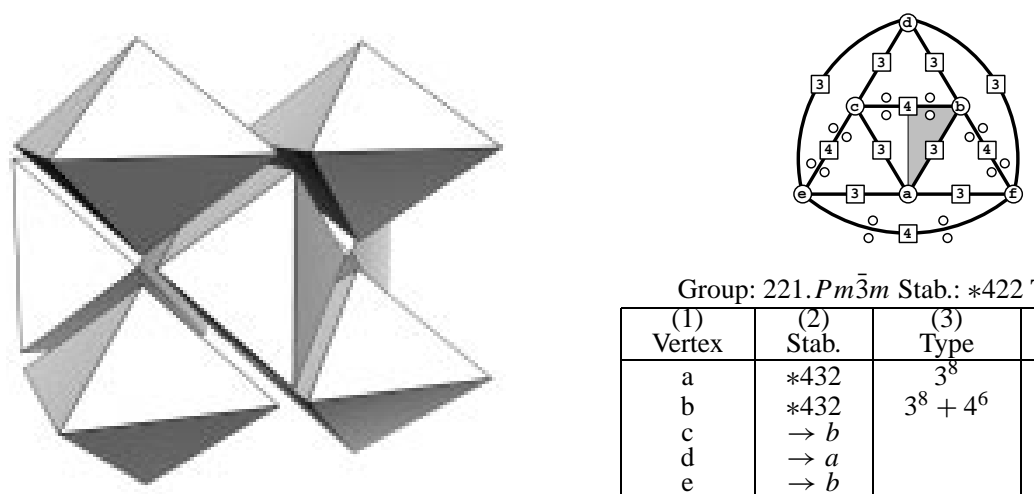

Group: 221.P $m \overline{3} m$ Stab.: $* 422$ Types: 43

\begin{tabular}{|c|c|c|c|}
\hline$(1)$ & $(2)$ & $(3)$ & $(4)$ \\
Vertex & Stab. & Type & Coord. \\
\hline $\mathrm{a}$ & $* 432$ & $3^{8}$ & $1,-1,1$ \\
$\mathrm{~b}$ & $* 432$ & $3^{8}+4^{6}$ & $0,0,0$ \\
$\mathrm{c}$ & $\rightarrow b$ & & $0,-2,0$ \\
$\mathrm{~d}$ & $\rightarrow a$ & & $1,-1,-1$ \\
$\mathrm{e}$ & $\rightarrow b$ & & $2,-2,0$ \\
$\mathrm{f}$ & $\rightarrow b$ & & $2,0,0$ \\
\hline
\end{tabular}

Fig. 12. OT1. 

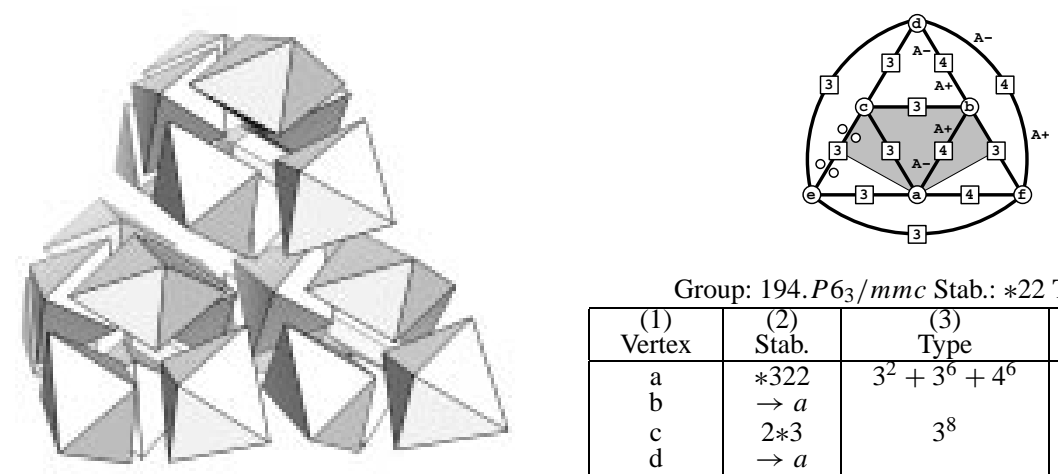

\begin{tabular}{|} 
Group: $194 . P 6_{3} / m m c$ Stab.: $* 22$ Types: 12 \\
\begin{tabular}{|c|c|c|c|}
\hline$(1)$ & $(2)$ & $(3)$ & $(4)$ \\
Vertex & Stab. & Type & Coord. \\
\hline $\mathrm{a}$ & $* 322$ & $3^{2}+3^{6}+4^{6}$ & $-1,1,-3$ \\
$\mathrm{~b}$ & $\rightarrow a$ & & $-1,3,1$ \\
$\mathrm{c}$ & $2 * 3$ & $3^{8}$ & $0,0,0$ \\
$\mathrm{~d}$ & $\rightarrow a$ & & $-3,-1,1$ \\
$\mathrm{e}$ & $\rightarrow c$ & & $-2,-2,-2$ \\
$\mathrm{f}$ & $\rightarrow a$ & & $-5,-1,-3$ \\
\hline
\end{tabular}
\end{tabular}

Fig. 13. OT2.
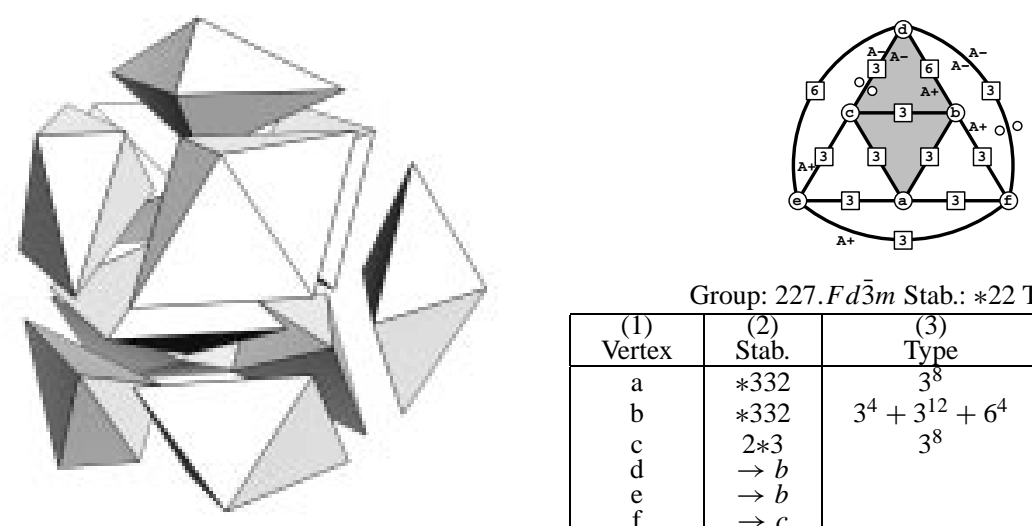

\begin{tabular}{|c|c|c|c|}
\multicolumn{5}{|c}{ Group: $227 . F d \overline{3} m$ Stab.: $* 22$ Types: 3} \\
\hline Vertex & Stab. & Type & Coord. \\
\hline $\mathrm{a}$ & $* 332$ & $3^{8}$ & $-1,-1,-1$ \\
$\mathrm{~b}$ & $* 332$ & $3^{4}+3^{12}+6^{4}$ & $-3,1,1$ \\
$\mathrm{c}$ & $2 * 3$ & $3^{8}$ & $0,0,0$ \\
$\mathrm{~d}$ & $\rightarrow b$ & & $-1,3,-1$ \\
$\mathrm{e}$ & $\rightarrow b$ & & $1,1,-3$ \\
$\mathrm{f}$ & $\rightarrow c$ & & $-2,0,-2$ \\
\hline
\end{tabular}

Fig. 14. OT3.
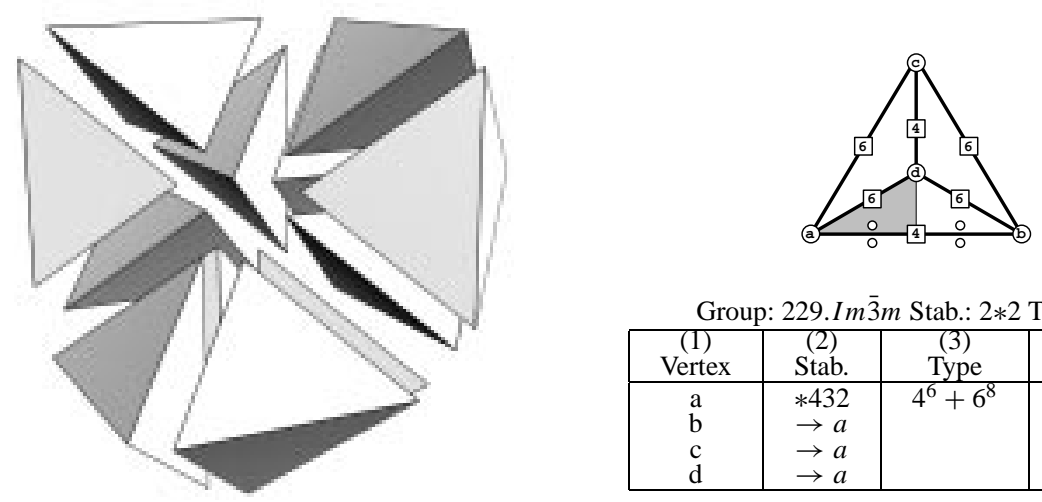

Group: $229 . \operatorname{Im} \overline{3} m$ Stab.: $2 * 2$ Types: 24

\begin{tabular}{|c|c|c|c|}
\hline$(1)$ & $(2)$ & $(3)$ & $(4)$ \\
Vertex & Stab. & Type & Coord. \\
\hline $\mathrm{a}$ & $* 432$ & $4^{6}+6^{8}$ & $0,0,0$ \\
$\mathrm{~b}$ & $\rightarrow a$ & & $0,0,2$ \\
$\mathrm{c}$ & $\rightarrow a$ & & $-1,-1,1$ \\
$\mathrm{~d}$ & $\rightarrow a$ & & $1,-1,1$ \\
\hline
\end{tabular}

Fig. 15. TT1. 

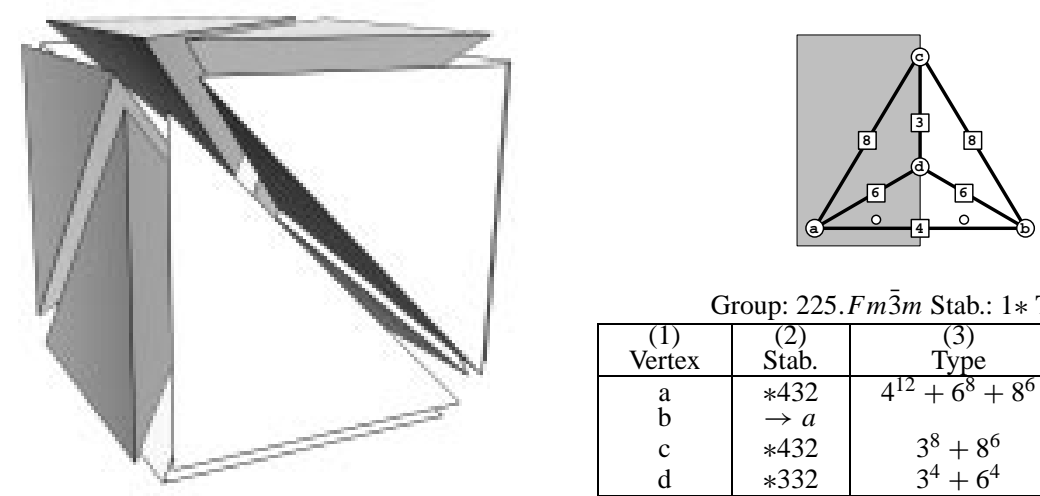

\begin{tabular}{|c|c|c|c|}
\multicolumn{4}{|c}{ Group: $225 . F m \overline{3} m$ Stab.: $1 *$ Types: 3} \\
\hline (1) & $(2)$ & $(3)$ & $(4)$ \\
Vertex & Stab. & Type & Coord. \\
\hline $\mathrm{a}$ & $* 432$ & $4^{12}+6^{8}+8^{6}$ & $0,0,0$ \\
$\mathrm{~b}$ & $\rightarrow a$ & & $0,-2,2$ \\
$\mathrm{c}$ & $* 432$ & $3^{8}+8^{6}$ & $0,0,2$ \\
$\mathrm{~d}$ & $* 332$ & $3^{4}+6^{4}$ & $-1,-1,1$ \\
\hline
\end{tabular}

Fig. 16. TT2.
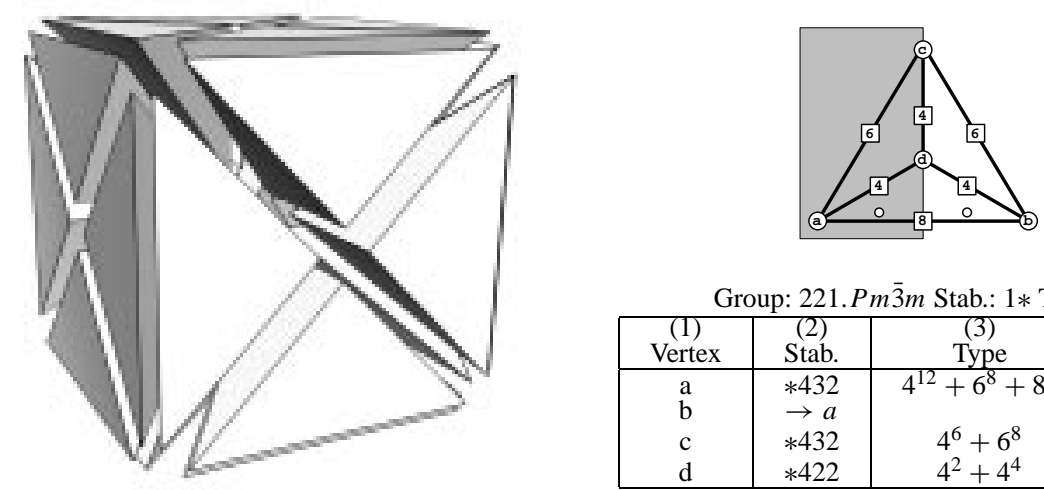

Group: $221 . P m \overline{3} m$ Stab.: $1 *$ Types: 5

\begin{tabular}{|c|c|c|c|}
\hline (1) & $(2)$ & $(3)$ & $(4)$ \\
Vertex & Stab. & Type & Coord. \\
\hline $\mathrm{a}$ & $* 432$ & $4^{12}+6^{8}+8^{6}$ & $0,0,0$ \\
$\mathrm{~b}$ & $\rightarrow a$ & & $2,0,0$ \\
$\mathrm{c}$ & $* 432$ & $4^{6}+6^{8}$ & $1,1,1$ \\
$\mathrm{~d}$ & $* 422$ & $4^{2}+4^{4}$ & $1,1,0$ \\
\hline
\end{tabular}

Fig. 17. TT3.
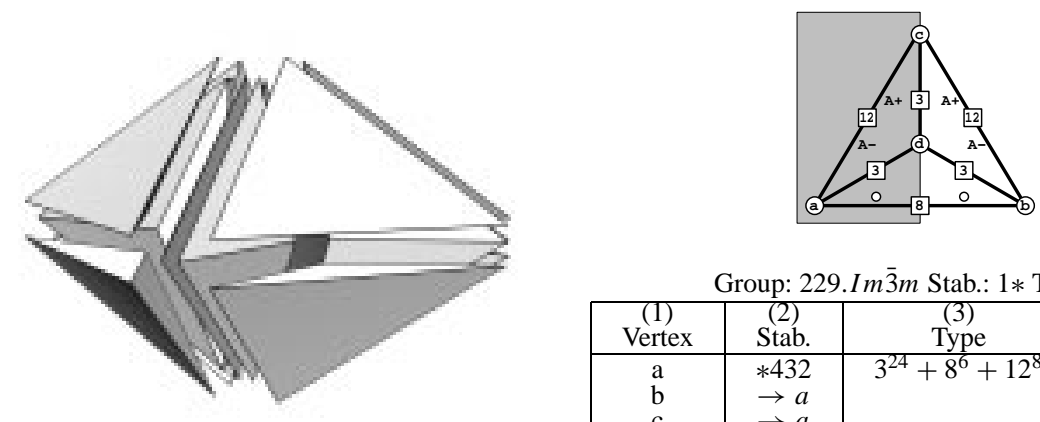

Group: $229 . \operatorname{Im} \overline{3} m$ Stab.: $1 *$ Types: 5

\begin{tabular}{|c|c|c|c|}
\hline (1) & $(2)$ & $(3)$ & $(4)$ \\
Vertex & Stab. & Type & Coord. \\
\hline $\mathrm{a}$ & $* 432$ & $3^{24}+8^{6}+12^{8}$ & $0,0,0$ \\
$\mathrm{~b}$ & $\rightarrow a$ & & $0,0,-4$ \\
$\mathrm{c}$ & $\rightarrow a$ & & $-2,2,-2$ \\
$\mathrm{~d}$ & $2 * 2$ & $3^{4}$ & $0,1,-2$ \\
\hline
\end{tabular}

Fig. 18. TT4. 

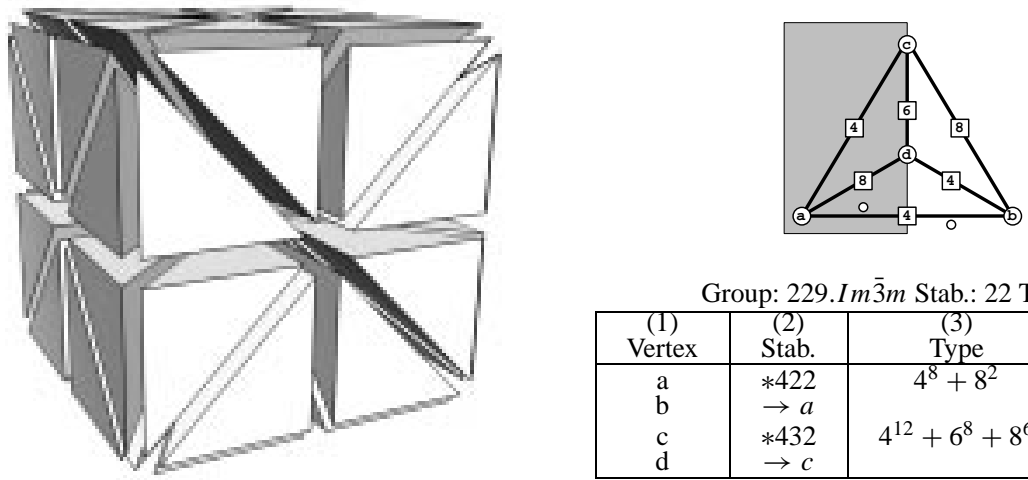

Group: $229 . \operatorname{Im} \overline{3} m$ Stab.: 22 Types: 5

\begin{tabular}{|c|c|c|c|}
\hline $\begin{array}{c}(1) \\
\text { Vertex }\end{array}$ & $\begin{array}{c}(2) \\
\text { Stab. }\end{array}$ & $\begin{array}{c}(3) \\
\text { Type }\end{array}$ & $\begin{array}{c}(4) \\
\text { Coord. }\end{array}$ \\
\hline $\mathrm{a}$ & $* 422$ & $4^{8}+8^{2}$ & $1,0,0$ \\
$\mathrm{~b}$ & $\rightarrow a$ & & $0,0,0$ \\
$\mathrm{c}$ & $* 432$ & $4^{12}+6^{8}+8^{6}$ & $0,1,0$ \\
$\mathrm{~d}$ & $\rightarrow c$ & & $1,0,-1$ \\
\hline
\end{tabular}

Fig. 19. TT5.
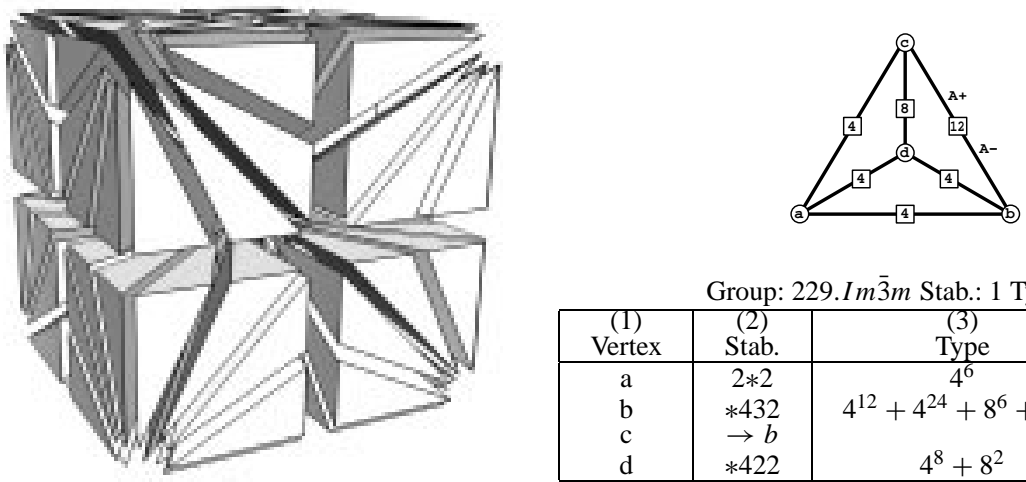

Group: $229 . \operatorname{Im} \overline{3} m$ Stab.: 1 Types: 1

\begin{tabular}{|c|c|c|c|}
\hline$(1)$ & $(2)$ & $(3)$ & $(4)$ \\
Vertex & Stab. & Type & Coord. \\
\hline $\mathrm{a}$ & $2 * 2$ & $4^{6}$ & $0,0,0$ \\
$\mathrm{~b}$ & $* 432$ & $4^{12}+4^{24}+8^{6}+12^{8}$ & $1,-3,1$ \\
$\mathrm{c}$ & $\rightarrow b$ & & $3,-1,-1$ \\
$\mathrm{~d}$ & $* 422$ & $4^{8}+8^{2}$ & $1,1,1$ \\
\hline
\end{tabular}

Fig. 20. TT6.
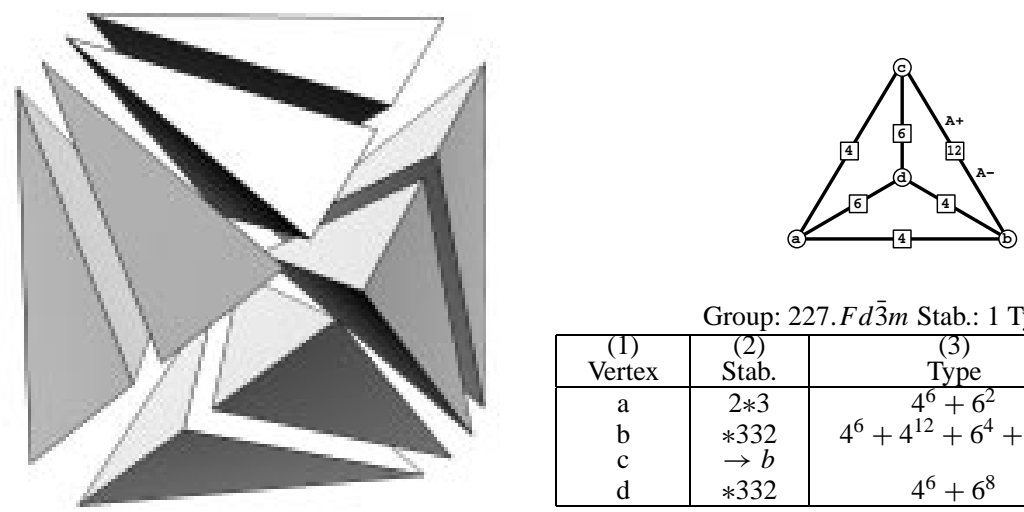

Group: 227. $F d \overline{3} m$ Stab.: 1 Types: 1

\begin{tabular}{|c|c|c|c|}
\hline$(1)$ & $(2)$ & $(3)$ & $(4)$ \\
Vertex & Stab. & Type & Coord. \\
\hline $\mathrm{a}$ & $2 * 3$ & $4^{6}+6^{2}$ & $0,0,0$ \\
$\mathrm{~b}$ & $* 332$ & $4^{6}+4^{12}+6^{4}+12^{4}$ & $1,-1,-1$ \\
$\mathrm{c}$ & $\rightarrow b$ & & $1,1,-1$ \\
$\mathrm{~d}$ & $* 332$ & $4^{6}+6^{8}$ & $1,1,1$ \\
\hline
\end{tabular}

Fig. 21. TT7. 

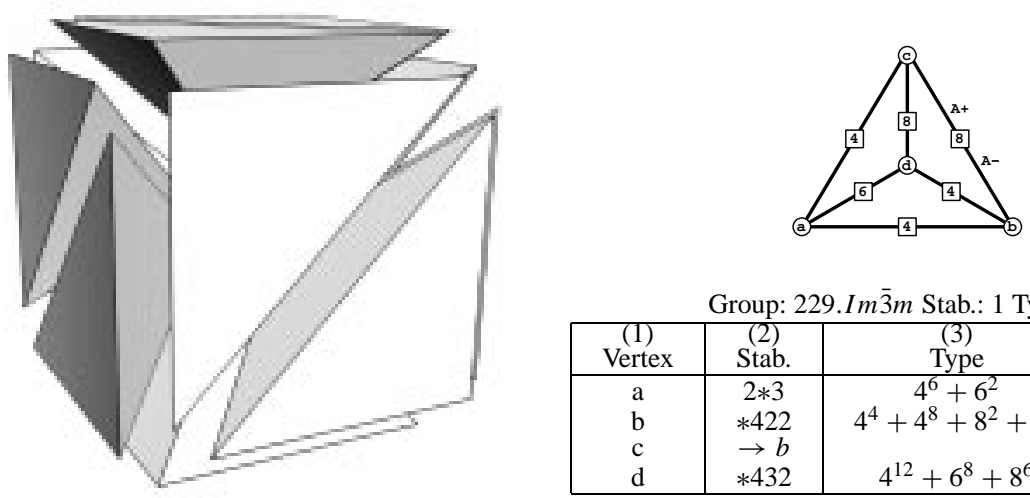

\begin{tabular}{|c|c|c|c|}
\multicolumn{4}{c}{ Group: $229 . \operatorname{Im} \overline{3} m$ Stab.: 1 Types: 1} \\
\hline$(1)$ & $(2)$ & $(3)$ & $(4)$ \\
Vertex & Stab. & Type & Coord. \\
\hline $\mathrm{a}$ & $2 * 3$ & $4^{6}+6^{2}$ & $0,0,0$ \\
$\mathrm{~b}$ & $* 422$ & $4^{4}+4^{8}+8^{2}+8^{4}$ & $2,-1,0$ \\
$\mathrm{c}$ & $\rightarrow b$ & & $0,1,-2$ \\
$\mathrm{~d}$ & $* 432$ & $4^{12}+6^{8}+8^{6}$ & $0,1,0$ \\
\hline
\end{tabular}

Fig. 22. TT8.

and Types: the number of different equivariant types contained in the topological family. This is followed by a table in which for each vertex labeled $a, b, c, \ldots$ in the diagram (1), we list its stabilizer group (2), its vertex type (as defined below) (3), and coordinates (4) that give rise to the depicted straight-edge realization. If a vertex labeled $j$ is equivalent to a vertex labeled $i$ already listed, then we write $\rightarrow i$ in column (2).

A vertex $v$ is of vertex type $p_{1}^{q_{1}}+p_{2}^{q_{2}}+p_{3}^{q_{3}}+\cdots$ if $v$ is incident to precisely $q_{i}$ equivalent edges of degree $p_{i}$ (with respect to the full combinatorial automorphism group of the vertex figure).

Comments. Types CT2, CT7, and CT9 can all be derived from the well-known tiling by rhombic dodecahedra (see [W]) by splitting each rhombic dodecahedron $D$ into four congruent topological cubes, using the center of $D$ as a new vertex. If we fix the spatial orientation of $D$, then there are precisely two different ways to split $D$. If all dodecahedra are split in the same way, then we obtain CT2, whereas in the two other cases each dodecahedron has precisely four neighbors that are split in the same way. Splitting layers of dodecahedra alternately gives rise to CT7, whereas CT9 is obtained by
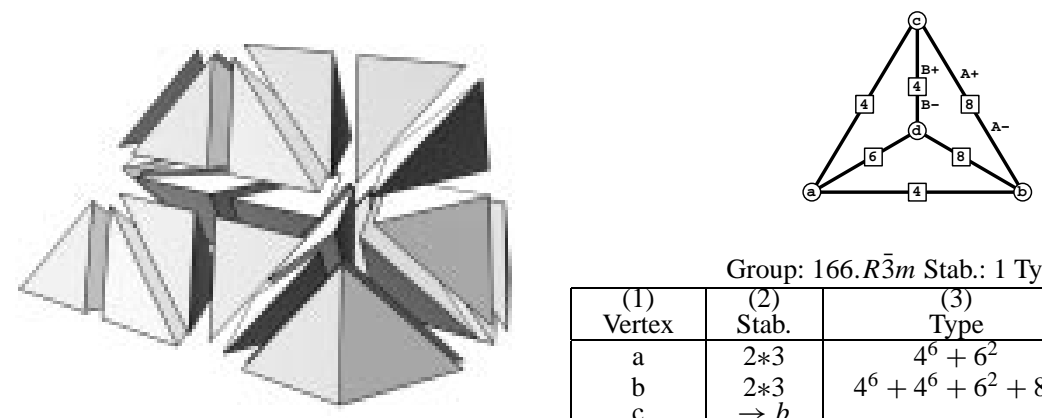

\begin{tabular}{|c|c|c|c|}
\multicolumn{4}{c}{ Group: $166 . R \overline{3} m$ Stab.: 1 Types: 1} \\
\hline $\begin{array}{c}(1) \\
\text { Vertex }\end{array}$ & $\begin{array}{c}(3) \\
\text { Stab. }\end{array}$ & Type & Coord. \\
\hline a & $2 * 3$ & $4^{6}+6^{2}$ & $0,0,0$ \\
b & $2 * 3$ & $4^{6}+4^{6}+6^{2}+8^{6}$ & $1,-1,1$ \\
c & $\rightarrow b$ & & $-1,-1,1$ \\
d & $\rightarrow b$ & & $1,1,1$ \\
\hline
\end{tabular}

Fig. 23. TT9. 
using yet another pattern of splitting. Tilings CT3 and CT4 are based on two-dimensional tilings. Type CT5 is obtained from TT1 by splitting each tetrahedron into four congruent topological cubes, using original face and tile centers as new vertices. We may regard CT6 as a (not face-to-face) tiling by triangular prisms. Type OT1 (or OT3) can be derived from the cube tiling CT1 (or CT2, respectively) by constructing a bipyramid over each face, using the centers of the adjacent cubes as apices. In OT2, three octahedra meet at a common edge joining with three screw-rotated copies to form a "hexagonal skew prism." These are stacked into "infinite hexagonal prisms," which fill space in a regular hexagon pattern. To obtain TT1, each bypiramid of OT1 is split into four tetrahedra along the line connecting the two apices. Type TT1 gives rise to TT3 by splitting each tile along a mirror plane and to TT4 by subdividing each tile into four, using the tile center as a new vertex. Type TT7 is obtained from TT1 by splitting each original tile into two new tetrahedra along a twofold symmetry axis not lying on a mirror plane. Barycentric subdivision of CT1 produces TT5, which also is a subdivision of TT3. We obtain TT6 and TT8 by considering the two possible ways of splitting tiles of TT5 into two new tetrahedra along a twofold symmetry axis. Types TT2 and TT8 have congruent tiles, but differ by rotations of certain quadruples of tiles by $\pi / 2$. Type TT9 can be regarded as a decomposition of a tiling by triangular prisms, in which each prism is split into six congruent tetrahedra.

By detailed comparison, our results confirm the classification of 298 equivariant types of fundamental tilings in topological family $\mathrm{CT} 1$ in $[\mathrm{P}]$. We obtain precisely the same classification of tile-transitive tilings of euclidean space by tetrahedra as contained in [MP1] and [MP2]. The tilings in our classification that have the additional property of being face-transitive coincide with those enumerated in $[\mathrm{DHM}]$ and the following four topological families occur there: CT1 $\sim 1$, CT2 $~ 13$, OT1 $\sim 9$, and TT1 10 .

\subsection{Which Convex Tetrahedra Tile Space?}

As mentioned above, the cube is the only regular platonic solid that tiles euclidean space. Aristotles wrongly asserted that the regular tetrahedron also does. This lead to confusion in the past. Thus, the question "Which tetrahedra fill space?" has received much mathematical attention [Se]. The classification of tile-transitive tilings by topological tetrahedra can be used to give an answer in the tile-transitive case.

For this purpose, we consider all possible convex realizations of each of the 46 equivariant types. Using methods described in [De], one can determine the group of linear components of the involved symmetries and thus formulate and solve a system of linear equations for the vertex positions of a tile. Examination of the arising solution spaces can be simplified by the following fact: If $\left(\mathcal{T}^{\prime}, \Gamma^{\prime}\right)$ is derived by symmetry breaking from $(\mathcal{T}, \Gamma)$, and if the dimension of the solution space for $\left(\mathcal{T}^{\prime}, \Gamma^{\prime}\right)$ is the same as for $(\mathcal{T}, \Gamma)$, then symmetry breaking can only be "realized" by bending edges or faces, i.e., by using nonconvex tiles. Application of this fact reduces the number of equivariant types of tilings to be examined to eleven, namely, the nine maximal representatives for each family and, in addition, two symmetry breakings of TT1.

By examination, tiling types TT1-TT8 have unique convex realizations up to similarity. Tilings TT2 and TT8, though topologically different, have identical tiles. Type 
TT9 has a one-parameter family of realizations, which is obtained by scaling along the vector $(1,1,1)$, the direction of an edge of degree 6 (see Figure 23). The first of the two symmetry breakings of TT1, which we denote by TT1' , leads to a second one-parameter family, again by scaling, e.g., along the vector $(1,1,1)$, the direction of an edge of degree 6 (see Figure 15). The second symmetry breaking of TT1 is a symmetry breaking of TT1' with a solution space of one dimension higher. However, the additional degree of freedom results from the fact that the whole tiling can be rotated around an axis of direction $(1,1,1)$ without changing the linear components of the symmetries, thus the convex realizations are the same as for TT1'.

In [Se] the derivation of space-filling convex tetrahedra by splitting certain classes of triangular prisms, as worked out by Sommerville [So1], [So2], is discussed. The author states that "all known" such tetrahedra can be obtained from Sommerville's four families of prisms. These, as is easy to see, lead to just two families of space-filling tetrahedra, which in fact correspond to our types TT1 and TT1'. Moreover, it turns out that TT9 can be obtained from TT1', and TT2-TT7 can be obtained from TT1 by "splitting" tetrahedra (see [Se] for a discussion of this concept).

We conclude that there exist precisely seven singular and two infinite families of convex tetrahedra that tile space tile-transitively. All such tetrahedra can be obtained by splitting triangular prisms. Moreover, these results imply that the collection of spacefilling convex tetrahedra discussed in $[\mathrm{Se}]$ is complete in the case of tile-transitive tilings.

\section{The General Approach}

In this section we introduce a general approach to classification problems of the kind treated in this paper. It is not restricted to platonic solids, e.g., in principle it can be used for any type of three-dimensional polyhedron, and it is not restricted to tile-transitive tilings, i.e., it can be easily adapted to enumerate tilings with more than one class of tiles, as we will show in a future paper.

\subsection{Outline of the Algorithm}

Given a topological polyhedron, all possible equivariant types of tile-transitive tilings of three-dimensional euclidean space based on it can be generated in the following steps:

(A1) Enumerate all possible equivariant polyhedra of the given topological type.

(A2) For each such equivariant polyhedron, systematically enumerate all possible identifications of pairs of faces.

(A3) For each choice of face identifications, enumerate all possible choices of degrees for each type of edge.

(A4) For each such equivariant polyhedron with face identifications and edge degrees, decide the existence problem, i.e., determine whether there exists a corresponding tile-transitive tiling of $\mathbb{E}^{3}$.

(A5) Finally, if such a tiling exists, then solve the realization problem, i.e., construct a realization of it. 
Additionally, the isomorphism problem must be solved, i.e., ensure that only one representative for each equivariant type is generated and, moreover, a representative for each topological type must be determined.

\subsection{Delaney Symbols}

The theory of Delaney symbols, introduced by Dress [Dr1], [Dr2], provides a suitable combinatorial model for formulating, studying, and solving classification problems concerning periodic tilings of simply connected manifolds. A number of algorithms and programs are based on this approach, see, e.g., [Hu], [DDH], and [DH2].

Given a periodic tiling $(\mathcal{T}, \Gamma)$ of a connected $d$-manifold admitting a barycentric subdivision, it was shown in [Dr1] and [Dr2] that this gives rise to a chamber system $\mathcal{C}$ over the index set $I:=\{0,1, \ldots, d\}$. In other words, $\mathcal{C}$ is a $\Sigma$-set, where $\Sigma:=\Sigma_{I}:=$ $\left\langle\sigma_{0}, \ldots, \sigma_{d} \mid \sigma_{i}^{2}=1\right\rangle$ is the free Coxeter group generated by $d+1$ involutions. The action of $\Gamma$ induced on $\mathcal{C}$ commutes with $\Sigma$. Thus, the quotient $\mathcal{D}:=\Gamma \backslash \mathcal{C}$ is a well-defined $\Sigma$-set. With each element $D \in \mathcal{D}$ one associates the matrix $M(D):=\left(m_{i j}(D)\right)_{I \times I}$, with entries of the form $m_{i j}(D):=\min \left\{m \in \mathbb{N}^{+} \mid C\left(\sigma_{i} \sigma_{j}\right)^{m}=C, C \in D\right\}$. Moreover, each such matrix is a Coxeter matrix, i.e., we have $m_{i i}(D)=1, m_{i j}(D)=2$ if $|i-j|>1$, and $m_{i j}(D)=m_{j i}(D)$ for all $D \in \mathcal{D}$ and $i, j \in I$.

In this way, the Delaney symbol $(\mathcal{D}, M)$ associated with $(\mathcal{T}, \Gamma)$ is obtained. Dress established that any two periodic tilings (of simply connected manifolds) are equivariantly equivalent if and only if their Delaney symbols are isomorphic. Moreover, a tiling is maximal if and only if its Delaney symbol does not have a smaller realizable homomorphic image. In the two-dimensional case and in certain three-dimensional geometries including euclidean 3-space, it can be shown that a homomorphic image of a realizable Delaney symbol is always realizable (see [MS] and [De]). Moreover, for each Delaney symbol, there is a unique smallest homomorphic image. Thus, maximal tilings correspond to smallest homomorphic images and are unique for each topological family, which justifies our earlier claim that maximal tilings in $\mathbb{E}^{3}$ are equivariantly equivalent if and only if they are topologically equivalent. For a detailed discussion of the three-dimensional case, see [DHM].

\subsection{Combinatorial Formulation of the Algorithm}

First, we consider step (A1) of the algorithm. The tetrahedron, octahedron, cube, icosahedron, and dodecahedron give rise to $11,33,33,22$, and 22 different equivariant polyhedra, respectively. These can be obtained by systematic application of symmetry breaking, as discussed, for example, in [DH1].

In (A2), assume that we are given an equivariant topological polyhedron $\left(P, \Gamma_{P}\right)$, in terms of its two-dimensional Delaney symbol $(\mathcal{D}, M)$, i.e., a finite, transitive $\Sigma_{\{0,1,2\}}$-set $\mathcal{D}$ with maps $m_{01}, m_{12}$. To generate all possible face-identifications for the polyhedron, we consider all possible definitions of the involution $\sigma_{3}$ on $\mathcal{D}$ that commute with $\sigma_{0}$ and $\sigma_{1}$. (This latter requirement follows from $m_{03}(D)=m_{13}(D)=2$ for all $D \in \mathcal{D}$.) 
Then, in (A3), for every edge of the polyhedron (up to symmetry) we choose the number of incident tiles in all possible ways. In terms of Delaney symbols, this involves generating all possible definitions of the map

$$
m_{23}: \mathcal{D} \rightarrow \mathbb{N}^{+}
$$

Again, certain requirements should be taken into account. In particular, if we define

$$
r_{i j}(D):=\min \left\{m \in \mathbb{N}^{+} \mid D\left(\sigma_{i} \sigma_{j}\right)^{m}=D\right\},
$$

then the so-called crystallographic restriction for rotational orders in a crystallographic group implies $m_{23}(D)=v \cdot r_{23}(D)$ for some $v \in\{1,2,3,4,6\}$, see, e.g., [DHM].

In (A4), for a given Delaney symbol the (euclidean) existence problem must be solved, i.e., decide whether the symbol does indeed correspond to some periodic tiling of three-dimensional euclidean space. By careful application of symmetry breaking to the Delaney symbol, this can be reduced to the task of deciding whether the topological realization of a given three-dimensional triangulation is homeomorphic to the threedimensional torus [De]. In principle, this problem is algorithmically decidable using the concept of irreducible surfaces and methods for recognition of the 3-torus and 3-sphere [Hak1], [Hak2], [R], however, an implementation of this algorithm does not seem practical.

Recently, the first author has established [De] that in many cases this question can be solved by application of standard methods from group theory $\left[\mathrm{Sc}^{+}\right]$combined with simplification steps suggested by Haken [Hak3]. Although all cases considered in our investigation were decidable in this way, our method is not guaranteed to reach a decision and thus it is not an algorithm in the strict sense.

To solve the isomorphism problem, we define a canonical numbering of all Delaney symbols and then ensure that for each isomorphism class we only produce the element with the smallest number. To be precise, after every step of the algorithm we check whether the current partially defined Delaney symbol can be rearranged to produce a smaller number, in which case we backtrack.

Obviously, the number of possible Delaney symbols grows exponentially and it has proven crucial to check certain necessary conditions as early as possible in stages (A2) and (A3). One such condition is that the two-dimensional subsymbols of the partial three-dimensional Delaney symbol must be spherical [DHM]. A second condition is based on the concept of an orbifold graph [DH2]. For a given symmetry group, this graph represents the singularity skeleton of the associated orbifold [T]. This (partial) graph can be extracted from a (partial) Delaney symbol and compared with all different graphs arising from three-dimensional euclidean space groups, see [DH2] for details. At present our programs can deal with Delaney symbols with up to approximately 100 elements.

Finally, in (A5) a system of linear equations for the coordinates of the constituents of the tiling can be formulated. In simple cases, the number of parameters resulting from these necessary conditions is small and they can be easily adjusted to obtain a realization, using simple optimization methods. 


\section{Acknowledgments}

The figures were produced using the scientific visualization program GEOMVIEW [LMP $\left.{ }^{+}\right]$. The group theory package GAP $\left[\mathrm{Sc}^{+}\right]$supplied an ideal platform for the implementation of our method. Thanks to Ludwig Balke and Emil Molnár for helpful comments.

\section{References}

[C] J.H. Conway. The orbifold notation for surface groups. In Groups, Combinatorics and Geometry, pages 438-447. Cambridge University Press, Cambridge, 1992. London Mathematical Society Lecture Note Series, vol. 165.

[DDH] O. Delgado Friedrichs, A.W.M. Dress, and D.H. Huson. Tilings and symbols—a report on the uses of symbolic calculation in tiling theory. In Computer Algebra in Science and Engineering, pages 273286. World Scientific, Singapore, 1995.

[De] O. Delgado Friedrichs. Euclidicity Criteria for Three-Dimensional Branched Triangulations. Ph.D. thesis, University of Bielefeld, 1994.

[DH1] A.W.M. Dress and D.H. Huson. Heaven and hell tilings. Rev. Topologie Structurale, 17:25-42, 1991.

[DH2] O. Delgado Friedrichs and D.H. Huson. Orbifold triangulations and crystallographic groups. Period. Math. Hungar., 34(1-2):29-55, 1997.

[DHM] A.W.M. Dress, D.H. Huson, and E. Molnár. The classification of face-transitive 3d-tilings. Acta Cryst. Sect. A, 49:806-817, 1993.

[Dr1] A.W.M. Dress. Regular polytopes and equivariant tessellations from a combinatorial point of view. In Algebraic Topology, pages 56-72. Göttingen, 1984. SLN, vol. 1172.

[Dr2] A.W.M. Dress. Presentations of discrete groups, acting on simply connected manifolds. Adv. in Math., 63:196-212, 1987.

[Hah] T. Hahn, editor. International Tables for Crystallography, vol. A. Reidel, Dordrecht, 1983.

[Hak1] W. Haken. Verfahren zur Aufspaltung einer 3-Mannigfaltigkeit in irreduzible 3-Mannigfaltigkeiten. Math. Z., 76:427-467, 1961.

[Hak2] W. Haken. Über das Homöomorphieproblem der 3-Mannigfaltigkeiten. Math. Z., 80:89-120, 1962.

[Hak3] W. Haken. Three-manifolds and normal surfaces, 1992. Manuscript on a series of lectures held at Bielefeld University.

[Hu] D.H. Huson. The generation and classification of tile- $k$-transitive tilings of the euclidean plane, the sphere and the hyperbolic plane. Geom. Dedicata, 47:269-296, 1993.

$\left[\mathrm{LMP}^{+}\right]$S. Levy, T. Munzner, M. Phillips, C. Fowler, and N. Thurston. Geomview. The Geometry Center, University of Minnesota, Minneapolis, 1.5 edition, 1995.

[M] E. Molnár. Symmetry breaking of the cube tiling and the spatial chess board by d-symbols. Beitr. Algebra Geom., 35(2):205-238, 1994.

[MP1] E. Molnár and I. Prok. Classification of solid transitive simplex tilings in simply connected 3-spaces, part I. In Intuitive Geometry, Szeged (Hungary), pages 311-362, 1994. North-Holland, Amsterdam. Colloquia Mathematica Societatis János Bolyai, vol. 63.

[MP2] E. Molnár, I. Prok, and J. Szirmai. Classification of solid transitive simplex tilings in simply connected 3-spaces, part II. Metric realizations. To appear in Period. Math. Hungar.

[MS] W. Meeks and P. Scott. Finite group actions on 3-manifolds. Invent. Math., 86:287-346, 1986.

[P] I. Prok. The euclidean space has 298 fundamental tilings with marked cubes by 130 space groups. In Intuitive Geometry, Szeged (Hungary), pages 363-388, 1994. North-Holland, Amsterdam, Colloquia Mathematica Societatis János Bolyai, vol. 63.

[R] J.H. Rubinstein. An algorithm to recognize the 3-sphere. Proceedings of the International Congress of Mathematicians, (Zürich, 1994), vol. 1, pages 601-611, 1995.

$\left[\mathrm{Sc}^{+}\right]$M. Schönert et al. GAP-Groups, Algorithms, and Programming, 5th edn. RWTHLDFM, Aachen, 1995.

[Se] M. Senechal. Which tetrahedra fill space? Math. Mag., 54(5):227-243, 1981.

[Sm] J.V. Smith. Topochemistry of zeolites and related materials. 1. Topology and geometry. Chem. Rev, 
88:149-182, 1988.

[So1] D.M.Y. Sommerville. Division of space by congruent triangles and tetrahedra. Proc. Roy. Soc. Edinburgh, 43:85-116, 1923.

[So2] D.M.Y. Sommerville. Space-filling tetrahedra in Euclidean space. Proc. Edinburgh Math. Soc., 41:49-57, 1923.

[T] W.P. Thurston. Three Dimensional Geometry and Topology, vol. I. Princeton University Press, Princeton, 1997.

[V] E.B. Vinberg, editor. Geometry II. Springer-Verlag, Berlin, 1993. Encyclopaedia of Mathematical Sciences, vol. 29.

[W] R. Williams. The Geometrical Foundation of Natural Structure, a Source Book of Design. Dover, New York, 1979.

Received February 13, 1997, and in revised form September 17, 1997. 